\title{
Integration of an Echo Component in a Standard XECG System: A Modular Approach
}

\author{
MCJ de Wijs, SH Meij, SP Nelwan, NHJJ van der Putten \\ Thoraxcenter, ErasmusMc, Rotterdam, Netherlands
}

\begin{abstract}
The most frequently used echocardiographic test of myocardial viability is dobutamine stress echocardiography. Besides an adequate Echo machine, the echo laboratory must have basic equipment such as a 12 lead ECG recording system, non invasive blood pressure monitoring, and a precision intravenous delivery system for pharmacological stress testing. This paper describes the integration of these components into a modular system with standard interfaces for hardware and software.

In realizing such an application, we aimed to use as many as possible existing applications (databases, Cardio Control Workstation, Internet Explorer, DICOM viewer, Internet Information Services). We also had to interface the following devices: infusion pump, ECG recorder, Echo machine, NIBP recorder. The system is planned to be in use at the end of 2004.
\end{abstract}

\section{Introduction}

In the Thoraxcenter a stress echo (XECHO) is recorded by three assistants. The first operates the echo device, the second is needed for reading the recorder ECG and operating the dobutamine pump, and the last has to manually enter all values into a VT100 terminal system. This system is used to store the data into a database program (CLINT) [1]. Afterwards, the echo itself is evaluated and the results are added to CLINT. Together, these actions lead to an ECG on paper, while blood pressure, dobutamine, and other results are stored manually in CLINT. Echo's are stored on a PACS (Picture Archive System) and can be retrieved on a PC with the Curad Dicom Viewer [2].

This manifold approach (In Figure 1 a picture is shown with from left to right the echo device, the blood pressure device next to it a dobutamine pump, the ECG device and the monitor of the CLINT terminal) has been in use for over 10 years. . Recently, however, research into the possibility of updating and integrating all components into one PC based system has started. .

\section{Methods}

An overview is given in figure 2 describing the dataflow. The EPR is the Electronic Patient Record where all modules are incorporated The different modules will be described below.

\section{Biosignal recording}

A phased approach was done based upon the acquisition of the Cardio Control Workstation (CCW) from Welch\&Allyn, which is already used to record ecgs from bicycle and ergometer stress tests. The introduction of $\mathrm{CCW}$ created the possibility to simultaneously measure ECG, blood pressure, and other parameters. The integration was started with the blood pressure device, Accutorr 3 from Datascopes, which was not supported by $\mathrm{CCW}$. Therefore a new device driver was written that utilizes a RS232 communication port. This driver requests measurement data from Accutorr every few seconds, compares it with the old measurements, and if a new measurement is detected, new data is sent to CCW.

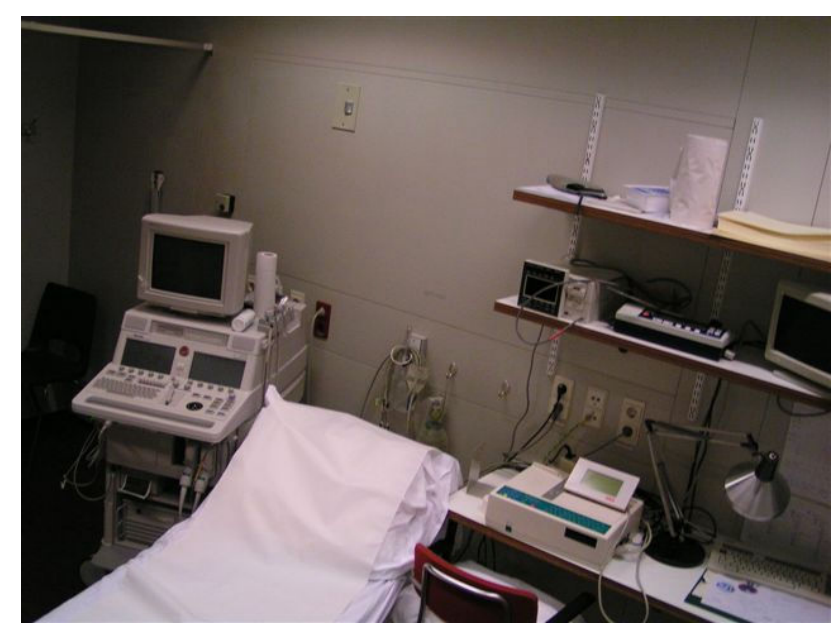

Figure 1: The old experimental setup

A different approach was necessary for the integration of the dobutamine pump interface. The pump can only be manually controlled; automatic infusion is not approved 
by medical protocols, hence a physician changes the infusion rate according to the patient's heart response to the perfusion (i.e. stage change). A special software module was built for integration into $\mathrm{CCW}$, and attached to the dobutamine pump by means of a second serial raw irDA infrared port. The module has its own device driver and is designed to simulate a manually operated treadmill.

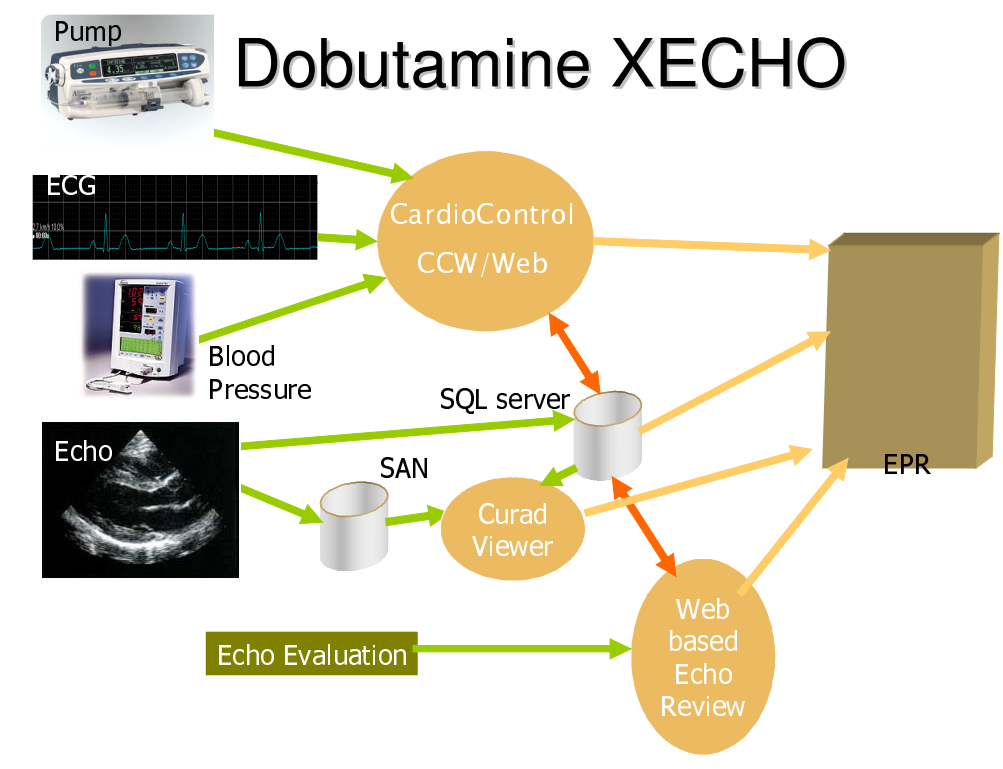

Figure 2: System Overview

The component is embedded into $\mathrm{CCW}$ as a child ActiveX component (Figure 3 ). Each change in infusion rate is interpreted as a stage change, which is inherited from the treadmill protocol. Stage change messages are sent to the program a keystroke (F9), accompanied by the updated values of both volume and drug infusion rate. In addition, these parameters are displayed on the module screen as a function of time. This way, ECG, blood pressure, as well as infusion rates and totals are automatically recorded and stored into the same database. The data from $\mathrm{CCW}$ can be reviewed from a web based summary page, which uses components from CCW for easy data access. These modules are called from an ASPX/C\# web page, which uses codes based upon pieces of the original source code from the $\mathrm{CCW}$ application to speed up development.

\section{Echo recording}

For the recording of the echoes, a Philips/HP device is used which stores the echo images on a SAN (Storage Area Network). Additionally, an index database is kept, which runs on the same server as the $\mathrm{CCW}$ database. This database is used by the DICOM viewer (Figure 4). By using this index database, the ECHO recording is linked and synchronized with the ECG recording, which can then be displayed on the web page. For each phase (rest, low dosage, peak dosage, recovery) 4 recordings are done (short axis, 4 chamber, 2 chamber, large axis) resulting in 16 images. This DICOM database can be queried for each image so it can be matched to an ECG on the web summary.

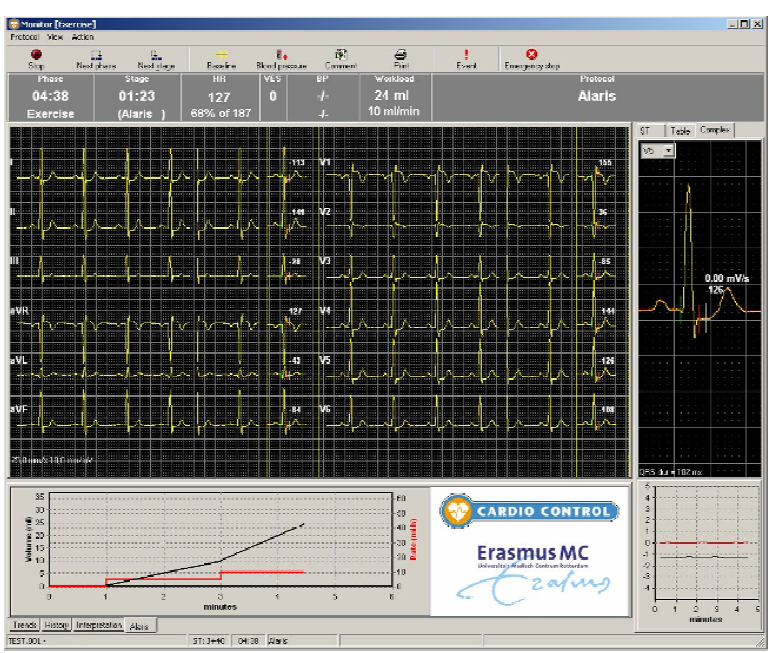

Figure 3: Simultaneous registration of XECG and dobutamine 


\section{Echo evaluation}

After the XECHO/ECG recordings the heart wall motion is evaluated (Figure 5). To this purpose the heart is divided into several sections (Table 1), each section is rated by a physician (Table 2 ).

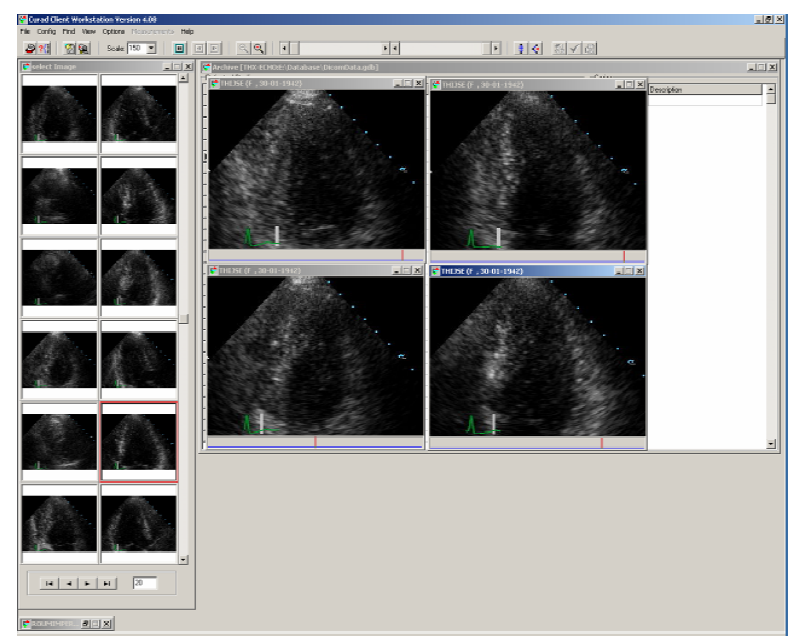

Figure 4: DICOM Viewer (Curad BV)

\begin{tabular}{|l|l|}
\hline Anterior & 7,2 \\
\hline Septum post & $11,10,5$ \\
\hline Septum ant & $12,6,1$ \\
\hline Lateral & $13,8,3$ \\
\hline Inferior & 9,4 \\
\hline Posterior & $14,15,16$ \\
\hline
\end{tabular}

Table 1 Heart Wall Sections and subsections according to the 17 segment ventricular wall division

\begin{tabular}{|l|l|}
\hline Normal & 1 \\
\hline Mild hypokinesias & 2 \\
\hline Severe hypokinesias & 3 \\
\hline Akinesia & 4 \\
\hline Dyskinesia & 5 \\
\hline Unknown & $\mathrm{X}$ \\
\hline
\end{tabular}

Table 2 Scores List

The scores for each section are used to calculate the WMS index[3], a measure of total wall motion. For an index of blood flow, the $L V E D V \mathrm{ml}, L V E S V \mathrm{ml}, L V$ $E F \%$, Peak $E$ velocity are calculated with the viewing software. The results of the evaluation can be filled out in an ASPX webpage with C\# server code, while the results of the $\mathrm{CCW}$ recording can be viewed on a sub page. The evaluation values are stored into the echo SQL database.

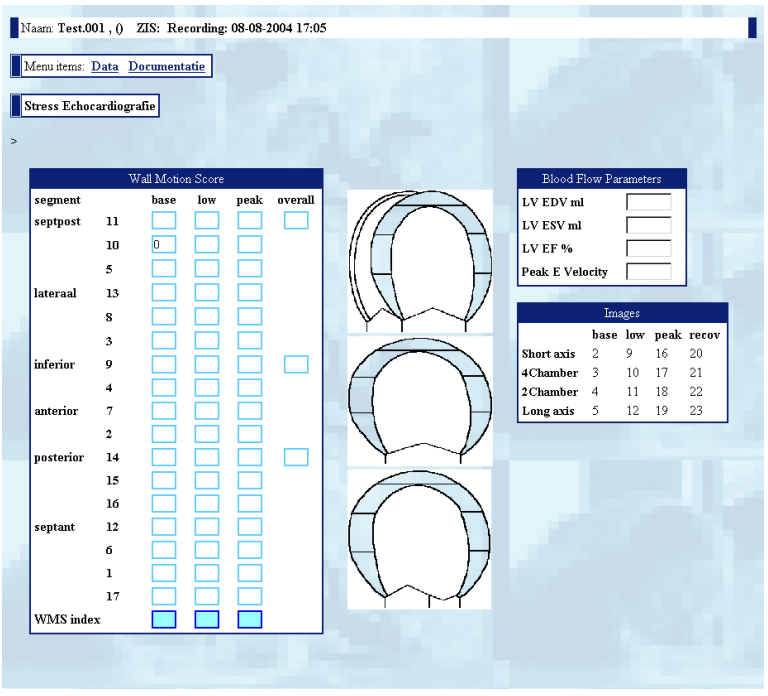

Figure 5: XECHO scoring component

\section{Results}

The ECG Summary webpage (Figure 6) contains all data related to dobutamine/blood pressure and graphically displays the start time of the first image per phase.

As described in the previous paragraph, the results consist of several web pages programmed in $\mathrm{C \#}$ and ASPX that as such can be displayed in Internet Explorer 5.5 or higher. Each page focuses on its primary application. Links with each other are created via the databases.

\section{Discussion and conclusions}

The Echo Evaluation form will change in the near future. It will be replaced by the Standardized Segmentation and Nomenclature as put forward by The American Heart Association [3]. When everything is embedded into the EPR the thumbnails in figure 6 will be clickable and functionality will be added to enable the viewing of the correct Echo in the DICOM viewer. Finally the ECG can be converted to DICOM and displayed synchronously with the Echo images. For research purposes, it still remains possible to export the data to CLINT because everything is stored in a SQL database.

\section{Acknowledgements}

Thanks go to Mr M. Lakamp and F. Kroon from Welch\&Allyn for their support and cooperation. 


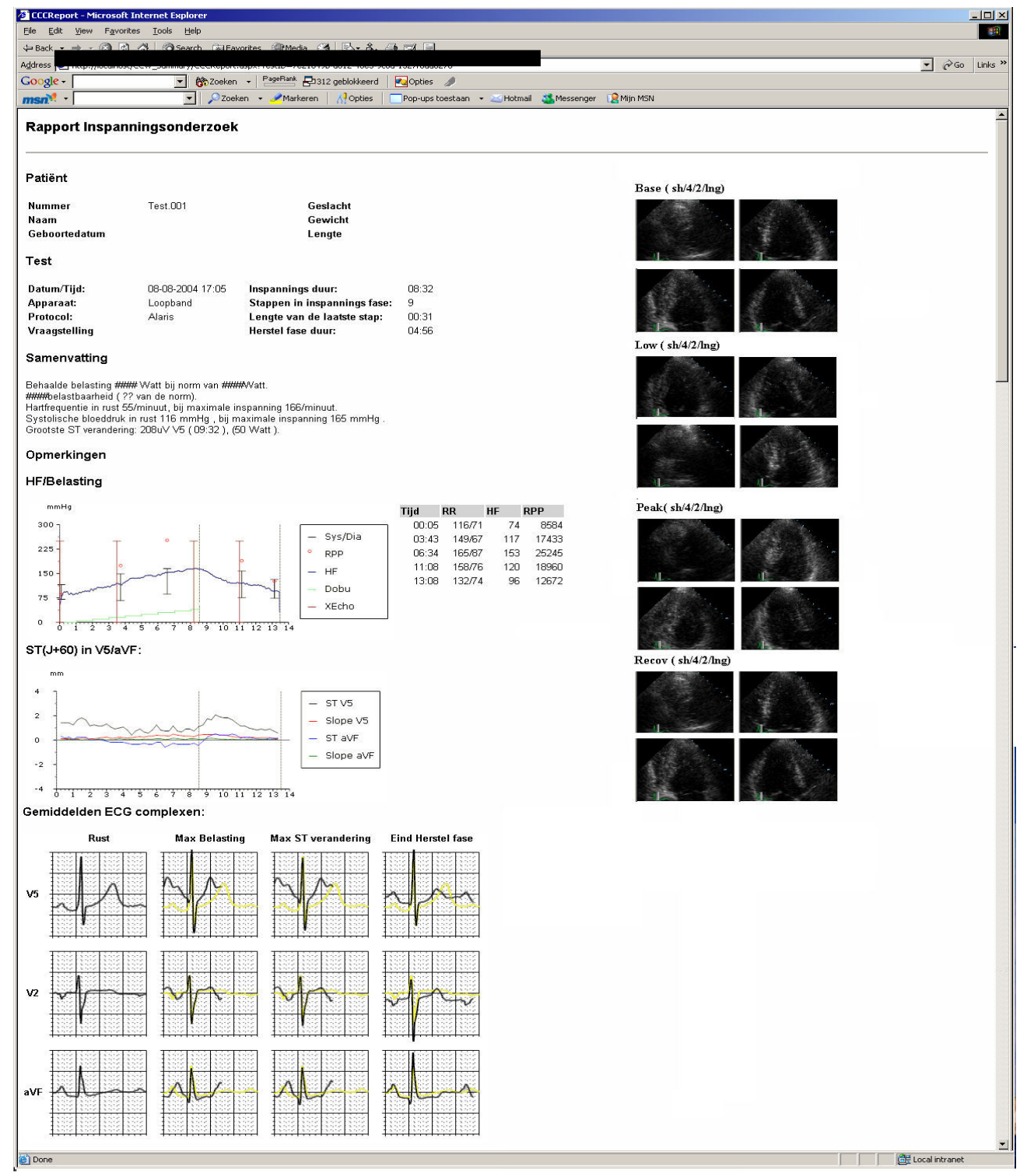

Figure 6: Example of Web Based Summary.

\section{References}

[1] Van Domburg RT,Zeelenberg C. CLINT: a clinical trial data management system. Proceedings of the 15 th annual meeting of the MUMPS Users'Group 1990:101-08.

[2] Van Der Putten N, Hamers R. Desktop Cardiography. Computers in Cardiology 2002;29;341-344

[3] Manuel D. Cerqueira et al. Standardized Myocardial Segmentation and Nomenclature for Tomographic Imaging of the Heart. AHA Scientific Statement
Address for correspondence

Marcel de Wijs

Thoraxcenter, room H549

ErasmusMc

Dr. Molenwaterplein 40

3015 GD Rotterdam, The Netherlands

M.deWijs@ErasmusMc.nl 